\title{
Esophageal and Gastric Cancer Pearl: a nationwide clinical biobanking project in the Netherlands
}

\author{
L. Haverkamp, ${ }^{1}$ K. Parry, ${ }^{1}$ M. I. van Berge Henegouwen, ${ }^{2}$ H. W. van Laarhoven, ${ }^{3}$ J. J. Bonenkamp, ${ }^{4}$ \\ T. M. Bisseling, ${ }^{5}$ P. D. Siersema, ${ }^{6}$ M. N. Sosef, ${ }^{7}$ J. H. Stoot, ${ }^{8}$ G. L. Beets, ${ }^{9}$ W. O. de Steur, ${ }^{10}$ \\ H. H. Hartgrink, ${ }^{10}$ H. W. Verspaget,${ }^{11}$ D. L. van der Peet, ${ }^{12}$ J. T. Plukker, ${ }^{13}$ B. van Etten,,${ }^{13}$ \\ B. P. L. Wijnhoven, ${ }^{14}$ J. J. van Lanschot, ${ }^{14}$ R. van Hillegersberg, ${ }^{1}$ J. P. Ruurda ${ }^{1}$
}

\begin{abstract}
${ }^{1}$ Departments of Surgery and ${ }^{6}$ Gastroenterology and Hepatology, University Medical Center Utrecht, Utrecht; ${ }^{2}$ Departments of Surgery and ${ }^{3}$ Medical Oncology, Academic Medical Center; ${ }^{11}$ Parelsnoer Institute;

${ }^{12}$ Department of Surgery, VU Medical Center, Amsterdam; ${ }^{4}$ Departments of Surgery and ${ }^{5}$ Gastroenterology and Hepatology, Radboud University Medical Center, Nijmegen; ${ }^{7}$ Department of Surgery, Atrium Medical Center Parkstad, Heerlen; ${ }^{8}$ Department of Surgery, Orbis Medical Center, Sittard; ${ }^{9}$ Department of Surgery, Maastricht University Medical Center, Maastricht; ${ }^{10}$ Department of Surgery, Leiden University Medical Center, Leiden; ${ }^{13}$ Department of Surgery, University Medical Center Groningen, Groningen; and ${ }^{14}$ Department of Surgery, Erasmus University Medical Center, Rotterdam, The Netherlands
\end{abstract}

SUMMARY. Esophageal and gastric cancer is associated with a poor prognosis since many patients develop recurrent disease. Treatment requires specific expertise and a structured multidisciplinary approach. In the Netherlands, this type of expertise is mainly found at the University Medical Centers (UMCs) and a few specialized nonacademic centers. Aim of this study is to implement a national infrastructure for research to gain more insight in the etiology and prognosis of esophageal and gastric cancer and to evaluate and improve the response on (neoadjuvant) treatment. Clinical data are collected in a prospective database, which is linked to the patients' biomaterial. The collection and storage of biomaterial is performed according to standard operating procedures in all participating UMCs as established within the Parelsnoer Institute. The collected biomaterial consists of tumor biopsies, blood samples, samples of malignant and healthy tissue of the resected specimen and biopsies of recurrence. The collected material is stored in the local biobanks and is encoded to respect the privacy of the donors. After approval of the study was obtained from the Institutional Review Board, the first patient was included in October 2014. The target aim is to include 300 patients annually. In conclusion, the eight UMCs of the Netherlands collaborated to establish a nationwide database of clinical information and biomaterial of patients with esophageal and gastric cancer. Due to the national coverage, a high number of patients are expected to be included. This will provide opportunity for future studies to gain more insight in the etiology, treatment and prognosis of esophageal and gastric cancer.

KEY WORDS: biobanking, esophageal and gastric cancer, the Netherlands.

Address correspondence to: Mr Jelle P. Ruurda, MD, PhD, University Medical Center Utrecht, Department of Surgery, PO BOX 85500, Utrecht, GA 3508, The Netherlands. Email: j.p.ruurda@umcutrecht.nl

The work described in this study was carried out in the context of the Parelsnoer Institute (PSI). PSI is part of and funded by the Dutch Federation of University Medical Centers and has received initial funding from the Dutch Government (from 2007-2011). Author's contributions: LH participated in the design of the study and wrote the manuscript, KP wrote the manuscript, PS participated in the design of the study and revised the manuscript critically, HL participated in the design of the study and revised the manuscript critically, MBH participated in the design of the study and revised the manuscript critically, JB participated in the design of the study and revised the manuscript critically, TB participated in the design of the study and revised the manuscript critically, JP participated in the design of the study and revised the manuscript critically, BE participated in the design of the study and revised the manuscript critically, HH participated in the design of the study and revised the manuscript critically, WS participated in the design of the study and revised the manuscript critically, DP participated in the design of the study and revised the manuscript critically, MS participated in the design of the study and revised the manuscript critically, JS participated in the design of the study and revised the manuscript critically, JJ participated in the design of the study and revised the manuscript critically, BW participated in the design of the study and revised the manuscript critically, RH participated in the design of the study and revised the manuscript critically and JR participated in the design of the study and revised the manuscript critically. All authors read and approved the final manuscript. 


\section{INTRODUCTION}

\section{Esophageal and gastric cancer}

The incidence of esophageal carcinoma is increasing in developed countries, whereas the incidence of gastric cancer has stabilized since its increase in the 1980s. The increase of esophageal cancer can mainly be attributed to the increase in adenocarcinoma of the esophagogastric junction among Caucasian males. ${ }^{1-4}$ Esophageal cancer is now the sixth and gastric cancer the second leading cause of cancerrelated mortality worldwide., ${ }^{3,4}$ The increased incidence of esophageal carcinoma was also reported in the Netherlands. ${ }^{5}$ In two decades (1989-2008), a threefold increase was seen for esophageal cancer, whereas the incidence of gastric cancer remained stable. ${ }^{5}$ The specific etiological factors that contributed to the dramatic increase and the prognosis of the disease are insufficiently elucidated.

Over the years, 5-year survival improved for esophageal cancer due to the increased use of neoadjuvant treatment and centralization of esophageal cancer surgery. However, 5-year survival remains poor. ${ }^{6-12}$ The recently conducted CROSS trial showed that the overall 5-year survival after chemoradiotherapy combined with surgery was $47 \%$ in the Netherlands for patients with esophageal cancer. ${ }^{13}$ For cardia and non-cardia gastric cancer, long-term survival rates have not improved over the past 20 years. ${ }^{14}$ The reported survival rates were $33 \%$ and $50 \%$, respectively, for cardia and non-cardia gastric cancer treated with surgery. ${ }^{14}$ Many patients with esophageal and gastric cancer develop metastatic disease or locoregional recurrence, and it remains difficult to predict which patients will develop recurrence disease.

Treatment of esophageal and gastric cancer requires a specific expertise and a structured multidisciplinary approach. This type of expertise is mainly found at the University Medical Centers (UMCs) and a few specialized nonacademic centers in our country. The UMCs have established a multidisciplinary collaboration to collect and store esophageal and gastric cancer biomaterial and to link this information to clinical data of the individual patients as part of the Parelsnoer Institute (PSI).

\section{History}

The PSI was established in 2007 by the Dutch Federation of UMCs (NFU) with its main goal to 'improve health by sharing science'. All eight UMCs of the Netherlands collaborated to achieve a nationwide database of clinical data and biomaterial for patients with selected medical conditions. Initially, eight different patients cohorts, the so-called 'pearls', were created by the PSI as seen in Table 1 . However, over the years more patient cohorts followed, and the
Table 1 Parelsnoer Institute

\begin{tabular}{|c|c|}
\hline Year & Pearl (patient cohort) \\
\hline \multirow[t]{8}{*}{2007} & Cerebrovascular accident \\
\hline & Type II diabetes mellitus \\
\hline & Hereditary colorectal cancer \\
\hline & $\begin{array}{l}\text { Inflammatory bowel diseases (Crohn's disease and } \\
\text { ulcerative colitis) }\end{array}$ \\
\hline & Leukemia \\
\hline & Neurodegenerative diseases \\
\hline & Rheumatoid arthritis \\
\hline & Renal failure \\
\hline 2010 & CONCOR (congenital heart disease) \\
\hline \multirow[t]{3}{*}{2011} & Multiple endocrine neoplasms \\
\hline & Ischemic heart disease \\
\hline & Pancreatic cancer and chronic pancreatitis \\
\hline \multirow[t]{2}{*}{2014} & Esophageal and gastric tumors \\
\hline & Parkinson \\
\hline
\end{tabular}

Esophageal and Gastric Cancer Pearl is now the 14th patient cohort that has started collecting data. The overall aim of the PSI was to realize a national infrastructure within the UMCs to collect clinical data and to set up a national biobank to collect biomaterial. The clinical data are linked to the biomaterial, and therefore it can be used to conduct more advanced research. This may ultimately lead to better health care in patients with the selected medical condition, partly because treatment is more personalized (for further information: http://www.parelsnoer.org).

\section{Objective}

The base of the Esophageal and Gastric Cancer Pearl is to implement a national infrastructure for research in patients with cancer of the esophagus and stomach. The scientific goal is to initiate and stimulate scientific research of high quality and of international importance. The main objective consists of three aims:

- To gain more knowledge in the etiology of esophageal and gastric cancer;

- To evaluate and improve the response on (neo)adjuvant and palliative treatment;

- To gain insight in the prognosis of esophageal and gastric cancer.

\section{STUDY DESIGN}

\section{Inclusion criteria and informed consent}

The protocols for this multicenter study were approved by the Institutional Review Board of the 
Table 2 Work flow

\begin{tabular}{|c|c|c|c|c|c|c|c|c|}
\hline & \multicolumn{7}{|c|}{ Time management } & \multirow[b]{2}{*}{ Executive } \\
\hline & T0 & $\mathrm{T} 1$ & $\mathrm{~T} 2$ & $\mathrm{~T} 3$ & $\mathrm{~T} 4$ & T5 & T6-T10 & \\
\hline Informed consent & $\mathrm{X}$ & - & - & - & - & - & - & $\mathrm{PhD}$ candidate / nurse practitioner \\
\hline Demographics & $\mathrm{X}$ & - & - & - & - & - & - & $\mathrm{PhD}$ candidate / nurse practitioner \\
\hline Medical history & $\mathrm{X}$ & - & - & - & - & - & - & $\mathrm{PhD}$ candidate / nurse practitioner \\
\hline Comorbidities & $\mathrm{X}$ & - & - & - & - & - & - & $\mathrm{PhD}$ candidate / nurse practitioner \\
\hline Life style behavior & $\mathrm{X}$ & - & - & - & - & - & - & $\mathrm{PhD}$ candidate / nurse practitioner \\
\hline Diagnosis-specific anamnesis & $\mathrm{X}$ & - & - & - & - & - & - & Specialist \\
\hline Screening physical examination & $\mathrm{X}$ & - & - & - & - & - & - & Specialist \\
\hline Collecting biopsies of suspected tumor & - & $\mathrm{X}$ & - & - & - & - & $\mathrm{X}$ & Endoscopist \\
\hline Collecting blood samples & - & $\mathrm{X}$ & - & - & - & $\mathrm{X}$ & - & Specialist \\
\hline Data neoadjuvant treatment & - & - & $\mathrm{X}$ & - & - & - & - & $\mathrm{PhD}$ candidate / nurse practitioner \\
\hline Collecting tissue samples & - & - & - & $\mathrm{X}$ & - & - & - & Specialist \\
\hline Surgical data & - & - & - & $\mathrm{X}$ & - & - & - & PhD candidate / nurse practitioner \\
\hline Data postoperative & - & - & - & - & $\mathrm{X}$ & - & - & $\mathrm{PhD}$ candidate / nurse practitioner \\
\hline Data adjuvant treatment & - & - & - & - & - & $\mathrm{X}$ & - & $\mathrm{PhD}$ candidate / nurse practitioner \\
\hline Follow-up & - & - & - & - & - & - & $\mathrm{X}$ & $\mathrm{PhD}$ candidate / nurse practitioner \\
\hline
\end{tabular}

T0, date of inclusion; T1, day of upper gastrointestinal endoscopy; T2, after confirmation of diagnosis and before surgery; T3, day of surgery; T4, 30 days after surgery; T5, several weeks after surgery; T6, one year after surgery; T7, two years after surgery; T8, three years after surgery; T9, four years after surgery; T10, five years after surgery.

Biobank of the University Medical Center Utrecht. Patients with (suspected) esophageal or gastric tumors are eligible for inclusion into the esophageal and gastric cancer pearl if examination takes place in one of the following eight UMCs: Academic Medical Center Amsterdam, Free University Medical Center Amsterdam, University Medical Center Utrecht, Leiden University Medical Center, Erasmus Medical Center Rotterdam, University Medical Center Groningen, University Medical Center Nijmegen and Maastricht University Medical Center (Atrium Medical Center Heerlen, Orbis Medical Center Sittard). Patients who undergo either an upper gastrointestinal endoscopy with the taking of biopsies to confirm the diagnosis and/or who undergo an esophagectomy or gastrectomy are included into the Esophageal and Gastric Cancer Pearl. Informed consent must be fully understood and signed prior to the inclusion. The informed consent covers encoded clinical data, biobanking, permission to approach the municipality register for mortality follow-up and permission to contact the general practitioner of the patient to obtain important data. The participants receive a copy of the signed informed consent.

\section{Data collection}

Clinical data entry is performed by the local coordinating investigator. The prospective clinical database is kept separately from the biobank with a secure method to link encoded clinical information to tissue samples. This database can exclusively be accessed by authorized personnel. Biomaterial is also collected and stored locally by the coordinating investigator. Table 2 and Figure 1 shows the workflow and flowchart of the study. The collected data contains the following items:

(C) 2015 International Society for Diseases of the Esophagus

\section{Demographics, patient's characteristics and physical examination}

During the first visit at the outpatient clinic of the participating hospital, information is gathered regarding the following items: gender, date of birth, family medical history, history of cancer-related illness or surgery, comorbidities, lifestyle behavior and drug use. Furthermore, a diagnosis-specific history is obtained (i.e. dysphagia, reflux disease, weight loss, etc.). Also, a screening physical examination is performed, including assessment of length and weight.

\section{Diagnostic workup}

All patients receive an upper gastrointestinal endoscopy. During endoscopy, biopsies of the suspected malignancy are taken to confirm the diagnosis of esophageal or gastric cancer and to retrieve biological characteristics of the tumor. If possible, an additional six biopsies are taken from the tumor to collect into the biobank. Immediately after excision, three of these samples are emersed in formalin in the endoscopy room. The other three biopsies are put in Eppendorf tubes with isopentane on dry ice to snap freeze at the endoscopy unit. At the department of pathology, the biopsies in formalin are embedded in paraffin and the biopsies in the Eppendorf tubes are directly put into the biobank freezers. Total time from endoscopic excision to fixation and snap freezing is kept to a minimum. Similarly, one paraffin biopsy and one snap freeze biopsy of normal mucosa are collected from the esophagus, the esophagogastric junction and from the stomach, if possible. The biopsies in paraffin are stored at room temperature and the snap-frozen biopsies at $-80^{\circ} \mathrm{C}$ in the biobank. 


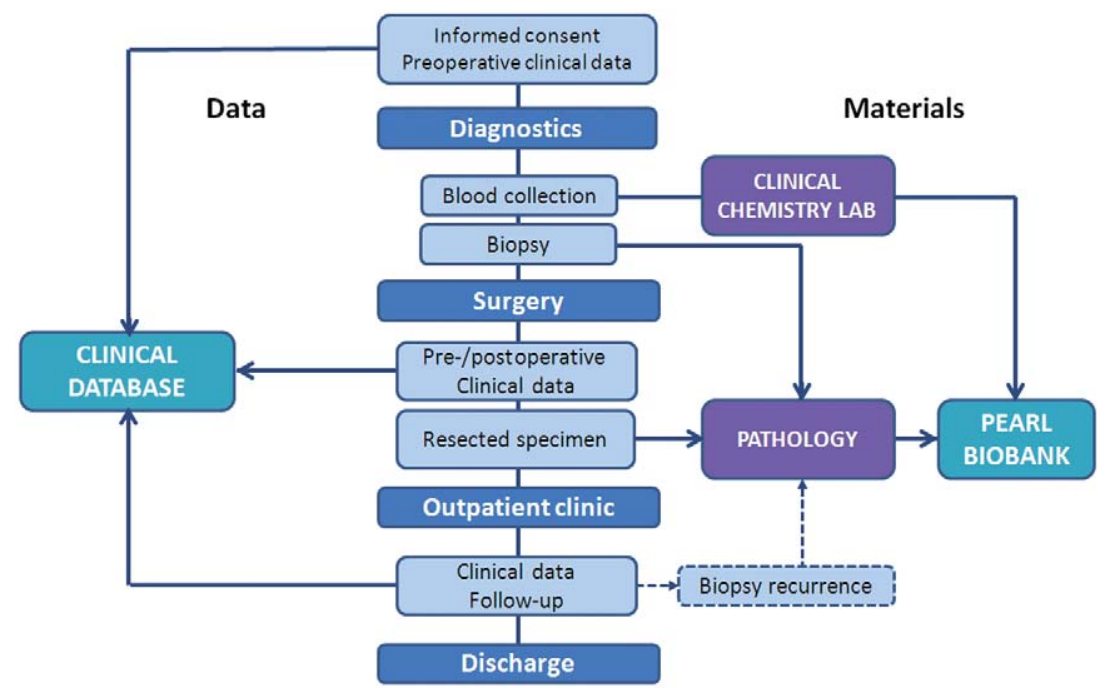

Fig. 1 Flowchart of Esophageal and Gastric Cancer Pearl.

\section{Blood samples}

Samples of venous blood (serum, EDTA plasma and EDTA blood for deoxyribonucleic acid [DNA] extraction) are collected preoperatively and postoperatively. The blood samples are processed and stored in certified biobank laboratories of the UMCs. EDTA plasma and serum samples are obtained from $10 \mathrm{~mL}$ blood and are stored in $0.5 \mathrm{~mL}$ aliquots at $-80^{\circ} \mathrm{C}$ in the biobank. EDTA blood samples for DNA extraction also consists of $10 \mathrm{~mL}$ blood, and after a quality control is performed the DNA, extracted within 4 weeks after venipuncture, is stored at $4^{\circ} \mathrm{C}$ or lower in the biobank. Maximum time for collecting, processing and storage of serum/plasma samples is 4 hours.

\section{Treatment}

If patients are eligible, they receive either neoadjuvant chemoradiation or perioperative chemotherapy depending on the site of the tumor and according to the Dutch upper gastrointestinal cancer guidelines. ${ }^{15}$ In case of a resectable tumor of the esophagus or stomach, patients undergo either an esophagus-cardia resection, partial or total gastrectomy or a total esophagogastrectomy. During surgery, the resection specimens are collected, and these are immediately transported in an unfixed state in a sterile closed box to the pathology department. At the department of pathology, the resection specimens are reviewed and if possible samples $\left(0.5 \mathrm{~cm}^{3}\right)$ of the tumor are removed to collect into the biobank without compromising diagnostic histopathological staging. Three of these samples are embedded in paraffin, and three are immediately snap frozen in isopentane on dry ice in order to maintain a good quality of DNA/RNA. The pathologist also collects additional samples of normal tissue of the esophagus, esophagogastric junction and stomach for storage in the biobank. Of each site, one sample is embedded in paraffin and one is snap frozen in isopentane. Total time from excision to snap freezing is estimated on 35 minutes. Information about the neoadjuvant treatment (chemotherapy or chemoradiotherapy) and the surgical procedure (type of resection, histopathological analysis) is entered in the prospective database.

\section{Postoperative data and follow-up}

Clinical data are collected until 30 days after surgery and consists of the following items: postoperative complications, mortality, readmission, radicality of resection, number of lymph nodes resected and stage according to the TNM staging system. ${ }^{16}$ In case a patient receives adjuvant treatment, information is entered in the database. Follow-up is maintained for a period of 5 years in the participating hospital. During these 5 years, data about local recurrences, distant metastasis, mortality and cause of death are collected annually. If biopsies of a suspected recurrence or metastasis are taken for histopathological confirmation, two additional biopsies are collected for storage in the biobank. One sample is embedded in paraffin, and one sample is snap frozen in isopentane.

\section{Biobanking}

All UMCs have their own storage for biomaterial, and together these storages form the national biobank for the Esophageal and Gastric Cancer Pearl. All materials are collected, identified, analyzed and stored in the biobank according to the standardized operating procedures established within the PSI to ensure homogenous sample (C) 2015 International Society for Diseases of the Esophagus 
Table 3 Collecting biomaterial

\begin{tabular}{|c|c|c|c|c|c|}
\hline Material & Amount & Frequency & Notification & Storage & Storage units \\
\hline \multicolumn{6}{|l|}{ Blood samples } \\
\hline 1 EDTA plasma & $1 \times 10 \mathrm{~mL}$ & $\begin{array}{l}1 \text { preoperative } \\
1 \text { postoperative }\end{array}$ & $\begin{array}{l}\text { Mandatory } \\
\text { Optional }\end{array}$ & $-80^{\circ} \mathrm{C}$ & $\begin{array}{l}\text { Preferably plasma } 5 \times \\
0.5 \mathrm{~mL} \text {, optional } \\
\text { blood/cell-pallet } 3 \times \\
0.9 \mathrm{~mL}\end{array}$ \\
\hline 2 Serum & $1 \times 10 \mathrm{~mL}$ & $\begin{array}{l}1 \text { preoperative } \\
1 \text { postoperative }\end{array}$ & $\begin{array}{l}\text { Mandatory } \\
\text { Optional }\end{array}$ & $-80^{\circ} \mathrm{C}$ & Preferably $5 \times 0.5 \mathrm{~mL}$ \\
\hline $\begin{array}{l}3 \text { EDTA blood sample } \\
\text { for DNA or pellet } \\
\text { from tube } 1\end{array}$ & $1 \times 10 \mathrm{~mL}$ & 1 preoperative & Mandatory & $4^{\circ} \mathrm{C}$ or $\leq 20^{\circ} \mathrm{C}$ & $\begin{array}{l}\geq 2 \text { aliquots DNA stock } \\
\text { solution }\end{array}$ \\
\hline \multicolumn{6}{|l|}{ Tissue samples } \\
\hline $\begin{array}{l}\text { Biopsies tumor / and } \\
\text { normal tissue } \\
\text { (esophagus, EGJ, } \\
\text { stomach) }\end{array}$ & $12 \dagger$ & 1 during endoscopy & Recommended & $\begin{array}{l}\text { Three paraffin and three } \\
\text { frozen samples from } \\
\text { normal tissue one each }\end{array}$ & \\
\hline Resection specimen & $6 \times 0.5 \mathrm{~cm}^{3}$ & 1 at surgery & Mandatory & $\begin{array}{l}\text { Preferably three paraffin } \\
\text { and three frozen samples }\end{array}$ & \\
\hline $\begin{array}{l}\text { Normal tissue } \\
\text { (esophagus, EGJ, } \\
\text { stomach) }\end{array}$ & $6 \times 0.5 \mathrm{~cm}^{3}$ & 1 at surgery & Mandatory & $\begin{array}{l}\text { One paraffin and one } \\
\text { frozen sample per site }\end{array}$ & \\
\hline $\begin{array}{l}\text { Biopsies } \\
\quad \text { recurrence/metastasis }\end{array}$ & 2 & Day of diagnostics & Recommended & $\begin{array}{l}\text { One paraffin and one } \\
\text { frozen sample }\end{array}$ & \\
\hline
\end{tabular}

$\lceil$ Only if possible. DNA, deoxyribonucleic acid; EDTA, Ethylenediaminetetraacetic acid; EGJ, esophagogastric junction.

collection. Each collected sample, as mentioned before and specified in Table 3, receives a unique sample code number, which is linked to the patient identification number through a biobank management information system software of the local biobank. Before clinical data linked to unique sample code numbers can be uploaded to the central database, the local patient's identification number is encrypted via a trusted third party by special software (Trusted Reversible Encryption Service ${ }^{\circ}$, Houten, Utrecht, The Netherlands) to respect the privacy of the donors and to ensure blinding of researchers to analysis. Hence, the central database contains encrypted clinical data linked to unique sample code numbers.

\section{Research}

All participating researchers can submit their study proposal to the scientific committee of the Esophageal and Gastric Cancer Pearl to conduct research with the clinical data and biomaterial gathered in the database. The scientific committee, consisting of two members of each UMC, judges the study proposal on the following criteria: the study will lead to novel insights regarding esophageal and gastric cancer, the study complies with requirements of an effective research methodology, the study will be performed by qualified investigators, the study is feasible and suitable. After acceptance of the research proposal by the scientific committee, the study can be conducted.

\section{Funding}

The founding of the PSI was co-financed by the Dutch Government, the NFU and the eight UMCs in the Netherlands. The continuation of the PSI is financed by the UMCs.

\section{DISCUSSION}

The biobank for the Esophageal and Gastric Cancer Pearl is designed to prospectively collect clinical data and biomaterial for research to gain more insight in its etiology, treatment and prognosis. In an era of increased interest in personalized medicine, the linkage of molecular data and genetic profiling to demographic, pathologic and clinical records will greatly enhance future studies of cancer etiology and risk factors. Moreover, the information about recurrences and survival status related to treatment therapies and genetic profiling will provide much information on progression and outcome of the disease.

Realizing their significance, biobanks have been established at national levels in many countries with strict regulations. For example, the General Practitioners Research Database in the UK was established to recruit more than half a million volunteers to collect blood and urine samples from 2006 to $2010 .^{17}$ These samples were linked to information obtained from questionnaires and physical information and have been valuable in many research topics concerning diabetes, major depression, osteoporosis and 
many more. ${ }^{18-20}$ Furthermore, the Esophageal and Gastric Center within the Academic Medical Center of Dublin has developed a repository of fresh frozen tissue, DNA, RNA and serum on patients with premalignant and malignant esophageal disease in $2004 .{ }^{21}$ They have collected samples of 420 patients in 6 years and used these samples for studies that included leptin and adiponectin receptor expression in esophageal cancer, ${ }^{22}$ prognostic significance of neuroepithelial transforming gene-1 in adenocarcinoma of the esophagogastric junction ${ }^{23}$ and more. Lastly, the Oesophageal Cancer Clinical and Molecular Stratification (OCCAMS) study group in the UK has started a similar program for tissue and data collection of patients with esophageal cancer across multiple specialist centers. They aimed to determine prognostic biomarkers and therapeutic targets for esophageal adenocarcinoma including whole genome sequencing. ${ }^{24,25}$ Also in the Netherlands, a national biobank has been established (PSI), which originally started collecting data of eight patient cohorts. It has now grown to 14 patient cohorts of selected medical conditions, and its growth is still expanding.

The Esophageal and Gastric Cancer Pearl is established to implement a national infrastructure for research in patients with cancer of the esophagus and stomach, which will allow us to gain more insight in the etiology and prognosis of esophageal and gastric cancer and to evaluate and improve the response on (neoadjuvant) treatment. The specific strength of the Esophageal and Gastric Cancer Pearl is the ability to compare and follow patients with different stages of esophageal and gastric cancer at a molecular and genetic level, which brings us a step closer to the identification of biomarkers for the diagnosis and progression of the disease. Furthermore, it allows us to study the sensitivity and resistance to neoadjuvant treatment. Prediction of pathologically complete response and nonresponse to neoadjuvant chemoradiotherapy by analysis of tissues and genetic profiling is of great clinical importance and could allow to decide what is the optimal treatment in each individual. Another clear strength of the Esophageal and Gastric Cancer Pearl is that healthy tissue is collected from the esophagus, esophagogastric junction and stomach. This will allow to directly compare acquired disease-related alterations within the genetic background of the patients.

The most important factor for achieving our goals is the implementation of a solid national research infrastructure that contains a standardized data collection and a solid long-term storage strategy. Due to the longitudinal character of research with biobank materials, it is necessary to ensure data harmonization over a long period of time. This will contribute to high-quality investigations for generations of researchers. We are realizing this by the use of the standard operating procedures to store biomaterial, which was released into the public domain by the Canadian Tumor Repository Network ${ }^{26}$ and by a secured method to link clinical information to tissue samples. Some considerations are raised concerning ethics regarding the collection of data. Before inclusion of the patients in this study, written informed consent is required. Furthermore, data entry is uploaded in an encoded manner, in order to ensure privacy of the patients as well as remaining the possibility to trace biological samples and data back to the original participant for linkage to follow-up. However, only exclusive personnel are authorized to access the secured code.

\section{PERSPECTIVES}

Thanks to the collaboration between the UMCs, the Esophageal and Gastric Cancer Pearl has a national coverage. Most of the patients with esophageal and gastric cancer are treated in UMCs in our country and therefore a high percentage of these patients will be included into the Esophageal and Gastric Cancer Pearl. The first patient was included in October 2014, after which data collection has started. The target aim is to include 300 esophageal and gastric cancer patients annually. With this great number of patients, promising results can be expected in the near future.

\section{CONCLUSION}

In conclusion, the eight UMCs of the Netherlands collaborate within the Parelsnoer Institute to establish a nationwide database of clinical information and biomaterial in patients with esophageal and gastric cancer. This collection of data will aid in gaining more insight in the etiology and prognosis of esophageal and gastric cancer and to evaluate and improve the response on (neoadjuvant) treatment.

\section{References}

1 Devesa S S, Blot W J, Fraumeni J F Jr. Changing patterns in the incidence of esophageal and gastric carcinoma in the United States. Cancer 1998; 83: 2049-53.

2 Bollschweiler E, Wolfgarten E, Gutschow C, Holscher A H. Demographic variations in the rising incidence of esophageal adenocarcinoma in white males. Cancer 2001; 92: 549-55.

3 Ferlay J, Shin H R, Bray F, Forman D, Mathers C, Parkin D M. Estimates of worldwide burden of cancer in 2008: GLOBOCAN 2008. Int J Cancer 2010; 127: 2893-917.

4 Jemal A, Siegel R, Ward E, Hao Y, Xu J, Thun M J. Cancer statistics, 2009. CA Cancer J Clin 2009; 59: 225-49.

5 Dikken J L, Lemmens V E, Wouters M W et al. Increased incidence and survival for oesophageal cancer but not for gastric cardia cancer in the Netherlands. Eur J Cancer 2012; 48: 1624-32.

6 Solaymani-Dodaran M, Logan R F, West J, Card T, Coupland C. Risk of oesophageal cancer in Barrett's oesophagus and gastro-oesophageal reflux. Gut 2004; 53: 1070-4. 
7 Wouters M W, Karim-Kos H E, le Cessie S et al. Centralization of esophageal cancer surgery: does it improve clinical outcome? Ann Surg Oncol 2009; 16: 1789-98.

8 Rudiger Siewert J, Feith M, Werner M, Stein H J. Adenocarcinoma of the esophagogastric junction: results of surgical therapy based on anatomical/topographic classification in 1,002 consecutive patients. Ann Surg 2000; 232: 35361.

9 Mariette C, Castel B, Toursel H, Fabre S, Balon J M, Triboulet J P. Surgical management of and long-term survival after adenocarcinoma of the cardia. Br J Surg 2002; 89: 1156-63.

10 Ito H, Clancy T E, Osteen R T et al. Adenocarcinoma of the gastric cardia: what is the optimal surgical approach? J Am Coll Surg 2004; 199: 880-6.

11 Mattioli S, Di Simone M P, Ferruzzi L et al. Surgical therapy for adenocarcinoma of the cardia: modalities of recurrence and extension of resection. Dis Esophagus 2001; 14: 104-9.

12 Coupland V H, Lagergren J, Luchtenborg M et al. Hospital volume, proportion resected and mortality from oesophageal and gastric cancer: a population-based study in England, 20042008. Gut 2013; 62: 961-6.

13 van Hagen $\mathrm{P}$, Hulshof M C, van Lanschot J J et al. Preoperative chemoradiotherapy for esophageal or junctional cancer. $\mathrm{N}$ Engl J Med 2012; 366: 2074-84.

14 Dassen A E, Dikken J L, van de Velde C J H, Wouters M W, Bosscha K, Lemmens V E. Changes in treatment patterns and their influence on long-term survival in patients with stages I-III gastric cancer in the Netherlands. Int J Cancer 2013; 133: 1859-66.

15 Integraal Kankercentrum Nederland, I. K. N. L. Oesofaguscarcinoom. Landelijke richtlijn, versie: 3.0. 2010.

16 Edge S B, Compton C C. The American Joint Committee on Cancer: the 7 th edition of the AJCC cancer staging manual and the future of TNM. Ann Surg Oncol 2010; 17: 1471-4.
17 Ollier W, Sprosen T, Peakman T. UK Biobank: from concept to reality. Pharmacogenomics 2005; 6: 639-46.

18 Harvey N C, Matthews P, Collins R, Cooper C, UK Biobank Musculoskeletal Advisory Group. Osteoporosis epidemiology in UK Biobank: a unique opportunity for international researchers. Osteoporos Int 2013; 24: 2903-5.

19 Smith D J, Nicholl B I, Cullen B et al. Prevalence and characteristics of probable major depression and bipolar disorder within UK biobank: cross-sectional study of 172,751 participants. PLoS ONE 2013; 8: e75362.

20 Tyrrell J S, Yaghootkar H, Freathy R M, Hattersley A T, Frayling T M. Parental diabetes and birthweight in 236030 individuals in the UK Biobank Study. Int J Epidemiol 2013; 42: 1714-23.

21 Ennis D P, Pidgeon G P, Millar N, Ravi N, Reynolds J V. Building a bioresource for esophageal research: lessons from the early experience of an academic medical center. Dis Esophagus 2010; 23: 1-7.

22 Howard J M, Cathcart M C, Healy L et al. Leptin and adiponectin receptor expression in oesophageal cancer. Br J Surg 2014; 101: 643-52.

23 Lahiff C, Schilling C, Cathcart M C et al. Prognostic significance of neuroepithelial transforming gene 1 in adenocarcinoma of the oesophagogastric junction. Br J Surg 2014; 101: 55-62.

24 Peters C J, Rees J R, Hardwick R H et al. A 4-gene signature predicts survival of patients with resected adenocarcinoma of the esophagus, junction, and gastric cardia. Gastroenterology 2010; 139: 1995-2004.

25 Weaver J M, Ross-Innes C S, Shannon N et al. Ordering of mutations in preinvasive disease stages of esophageal carcinogenesis. Nat Genet 2014; 46: 837-43.

26 Canadian Tumour Repository Network, CTR. Standard Operating Procedures, 2004. 